\title{
Tunneling Time and Weak Measurement in Strong Field Ionization
}

\author{
Tomáš Zimmermann, ${ }^{1,2}$ Siddhartha Mishra, ${ }^{1}$ Brent R. Doran, ${ }^{3}$ Daniel F. Gordon, ${ }^{4}$ and Alexandra S. Landsman ${ }^{1,2,5, *}$ \\ ${ }^{1}$ Seminar for Applied Mathematics, ETH Zurich, CH-8093 Zurich, Switzerland \\ ${ }^{2}$ Physics Department, ETH Zurich, CH-8093 Zurich, Switzerland \\ ${ }^{3}$ Department of Mathematics, ETH Zurich, CH-8093 Zurich, Switzerland \\ ${ }^{4}$ Radiation and Acceleration Physics Section, Naval Research Laboratory, Washington, D.C. 20375, USA \\ ${ }^{5}$ Max Planck Institute for the Physics of Complex Systems, D-01187 Dresden, Germany
}

(Received 18 July 2015; published 10 June 2016)

\begin{abstract}
Tunneling delays represent a hotly debated topic, with many conflicting definitions and little consensus on when and if such definitions accurately describe the physical observables. Here, we relate these different definitions to distinct experimental observables in strong field ionization, finding that two definitions, Larmor time and Bohmian time, are compatible with the attoclock observable and the resonance lifetime of a bound state, respectively. Both of these definitions are closely connected to the theory of weak measurement, with Larmor time being the weak measurement value of tunneling time and Bohmian trajectory corresponding to the average particle trajectory, which has been recently reconstructed using weak measurement in a two-slit experiment [S. Kocsis, B. Braverman, S. Ravets, M. J. Stevens, R. P. Mirin, L. K. Shalm, and A. M. Steinberg, Science 332, 1170 (2011)]. We demonstrate a big discrepancy in strong field ionization between the Bohmian and weak measurement values of tunneling time, and we suggest this arises because the tunneling time is calculated for a small probability postselected ensemble of electrons. Our results have important implications for the interpretation of experiments in attosecond science, suggesting that tunneling is unlikely to be an instantaneous process.
\end{abstract}

DOI: 10.1103/PhysRevLett.116.233603

How much time does it take for a particle to quantum tunnel through a potential barrier? This question has been a subject of intense theoretical debate for the last 80 years [1-6]. Time is not a quantum operator; hence, in contrast to tunneling probabilities, tunneling time itself is, famously, not a well-defined concept in quantum mechanics. Many different definitions have been proposed, and though defined and often invoked independently of a physical regime, they may actually be practically relevant only in different regimes; theoretical developments to date have shed little light on which is applicable when.

Experiments measuring tunneling of photons have found superluminal (although noninstantaneous) barrier propagation time $[7,8]$, which has been explained using ideas from weak measurement [9]. In strong field ionization, the bestknown proposal for experimentally measuring tunneling time is the attoclock [5]. Attoclock measurements recently found subluminal tunneling time in helium over a wide intensity range, using two independent experimental apparatuses [10]. Here, we bring weak measurement to bear for the first time in strong field ionization, with an eye towards these experiments.

Recently, weak measurement was used to reconstruct the average photon trajectory in a two-slit experiment, showing that these trajectories are Bohmian [11]. (A detailed theoretical discussion of the relationship between Bohmian mechanics and weak values can be found in [12].) Bohmian predictions have been found to agree in virtually every respect with the predictions of conventional quantum mechanics. Tunneling time is one notable example where conventional quantum mechanics offers a number of conflicting definitions, while Bohmian mechanics privileges one: namely, the time that the Bohmian trajectory spends between the entrance and exit points of the potential barrier. Here, we implement some of the best-known tunneling time definitions derived within conventional quantum mechanics, and we also compute the time that the Bohmian trajectory spends inside the barrier during strong field ionization. We find that Bohmian time is likely too large to correspond to tunneling time but, rather, agrees closely with the resonance lifetime of a bound state.

Our setting of strong field ionization of atoms is especially relevant for attosecond science experiments where tunnel ionization is regularly used to probe electron dynamics inside atoms and molecules on the attosecond time scale [13-15]. The interpretation of such experiments both heavily relies on the well-known tunneling model and, importantly, neglects the time delays associated with the tunneling process itself. Such delays are often assumed to be instantaneous or imaginary [16] since momentum is imaginary in the classically forbidden region, which in turn leads to imaginary time [17]. This argument, however, is applicable to tunneling in general [1]; hence, the need to explain how instantaneous (and therefore superluminal) tunneling would not violate physical causality still remains. Here, we find, however, that none of the well-known 
approaches to tunneling delays predict instantaneous tunneling, if a full solution, rather than a saddle-point approximation (such as that done in Ref. [17]), is used.

Prior work on tunneling time in strong field ionization [10] calculated four well-known tunneling time definitions (but not Bohmian time) for the strong field ionization of helium by using a short-range potential approximation, which neglects the long tail of the Coulomb potential, resulting in a triangular barrier of width $I_{p} / F$, where $I_{p}$ and $F$ are the ionization potential and the field strength, respectively. The problem was then solved in analogy to free propagation by matching a free wave outgoing solution with an experimental observable.

Arguably, for the purposes of calculating tunneling time, the bound state problem is fundamentally different from free propagation, and therefore it is desirable to fully account for the bound state wave function. Here, we fully account for the Stark-shifted ground state wave function of the atom and fully include the Coulomb field. Our solution is exact, in the optical tunneling limit of $\gamma \rightarrow 0$, for hydrogen and helium, within the validity of a single active electron approximation. [Here, $\gamma=\omega\left(2 I_{p}\right)^{1 / 2} / F$ is the Keldysh parameter, with $\omega$ being the frequency of the laser.] We find that, for the four tunneling time definitions (but not Bohmian time), the agreement between the exact solution and the short-range potential approximation, such as was implemented in Ref. [10], is very good, as long as the barrier width of the triangular barrier is similar to the exact barrier width.

These four tunneling times are based on very different models, but they can all be expressed in terms of the transmission amplitude $T=|T| e^{i \theta}$ [4], where $T=\psi\left(q_{1}\right) / \psi\left(q_{2}\right)$, and $\psi$ is the wave function value at the outer $\left(q_{1}\right)$ or inner $\left(q_{2}\right)$ classical turning point. Deriving $T$ with respect to the height of the potential $V$, and the incident energy of the particle $E$, one obtains Larmor (LM) time, $\tau_{\mathrm{LM}}$, the Büttiker-Landauer (BL) time, $\tau_{\mathrm{BL}}$, the Eisenbud-Wigner (EW) time, $\tau_{\mathrm{EW}}$, and the PollackMiller (PM) time, $\tau_{\mathrm{PM}}$ (for detailed discussions, see, for example, Refs. [3,6]). The first two times depend on the potential and have been called the resident (or dwell) time,

$$
\tau_{\mathrm{BL}}=-\hbar \partial \ln |T| / \partial V, \quad \tau_{\mathrm{LM}}=-\hbar \partial \theta / \partial V .
$$

The other two times depend on the incident energy of the particle and have been called the passage time,

$$
\tau_{\mathrm{PM}}=\hbar \partial \ln |T| / \partial E, \quad \tau_{\mathrm{EW}}=\hbar \partial \theta / \partial E .
$$

The Buttiker-Landauer time [2] and the Pollack-Miller time [18] depend on the probability of transmission, and they can therefore be well approximated using WKB. The Buttiker-Landauer time is actually closely related to the Keldysh time (see Ref. [6] for an explanation), while the Pollack-Miller time corresponds to the time average of the flux-flux correlation function [19]. The other two times-namely, the Larmor [20] and Eisenbud-Wigner times [21] — are phase dependent. This phase dependence makes the evaluation considerably more difficult, as the usual saddle-point and WKB approaches fail (see Ref. [6] for a discussion) and a complete solution of the transmission amplitude is necessary. The Larmor time was originally defined as given by the precession of the electron spin inside a rectangular magnetized barrier [20], but it has since been generalized to arbitrary barriers [22]. The Eisenbud-Wigner time is well known in single photon ionization (for a detailed treatment, see, for example, an excellent recent review by Pazourek et al. [15]).

In addition to the four definitions above, we also compute the Bohmian tunneling time, defined as the time which it takes a Bohmian trajectory to pass the region between the two classical turning points. In the Bohmian formalism, the wave function is expressed as $\psi(\vec{r}, t)=$ $R(\vec{r}, t) e^{i S(\vec{r}, t) / \hbar}$, where $R$ and $S$ are real valued. The Bohmian trajectory is determined by the probability density, given by $\rho(\vec{r}, t)=R(\vec{r}, t)^{2}$ and the velocity, given by $\vec{v}=\vec{\nabla} S / m=\vec{j}(\vec{r}, t) / \rho(\vec{r}, t)[23,24]$, where $\vec{j}=$ $(i / 2)\left(\psi \vec{\nabla} \psi^{*}-\psi^{*} \vec{\nabla} \psi\right)$ is the probability flux. Since flux is constant for solutions of the stationary Schrödinger equation, the Bohmian time is computed as

$$
\tau_{\text {Bohmian }}=\frac{1}{j} \int_{q_{1}}^{q_{2}} \rho d q .
$$

The Bohmian time, as well as the other times, is computed in the adiabatic limit by solving the Schrödinger equation for the hydrogen atom in the homogeneous electric field $F$ :

$$
\left(-\frac{\Delta}{2}+\frac{1}{r}-F z\right) \psi=E \psi
$$

The above equation is separable in the parabolic coordinates $\xi=r+z, \eta=r-z$, and $\phi=\arctan (y / x)$ [25]. Substituting $\psi(\xi, \eta, \phi)=(\xi \eta)^{-1 / 2} \chi_{1}(\xi) \chi_{2}(\eta) e^{-i m \phi}$ into Eq. (4), we obtain two one-dimensional equations for $\chi_{1}(\xi)$ and $\chi_{2}(\eta)$ :

$$
\begin{aligned}
& -\frac{1}{2} \frac{\partial^{2} \chi_{1}}{\partial \xi^{2}}+\left(\frac{m^{2}-1}{8 \xi^{2}}-\frac{\beta_{1}}{2 \xi}+\frac{F \xi}{8}\right) \chi_{1}=\frac{E}{4} \chi_{1}, \\
& -\frac{1}{2} \frac{\partial^{2} \chi_{2}}{\partial \eta^{2}}+\left(\frac{m^{2}-1}{8 \eta^{2}}-\frac{\beta_{2}}{2 \eta}-\frac{F \eta}{8}\right) \chi_{2}=\frac{E}{4} \chi_{2},
\end{aligned}
$$

which are coupled by the separation constants $\beta_{1}$ and $\beta_{2}$ satisfying $\beta_{1}+\beta_{2}=1$. Whereas all solutions of Eq. (5) are bound and the energy spectrum is discrete, Eq. (6) has unbound solutions with a continuous energy spectrum. The ionization is therefore described by Eq. (6), and the coordinate $\eta$ naturally corresponds to the tunneling degree 
of freedom. In order to describe the steady-state ionization process, one first has to find the solution of Eq. (6) which is outgoing for $\eta \rightarrow \infty$. Details of the procedure used to find the outgoing solution of Eq. (6) may be found in the Supplemental Material [26].

In addition to the exact solution of the Schrödinger equation (4), tunneling times were computed using the short-range potential approximation, as was previously done in Ref. [10]. The short-range potential is a consequence of the strong field approximation (SFA), originally introduced by Keldysh [27], and in the adiabatic limit reduces to the propagation through a static one-dimensional triangular barrier [28], given by Eq. (7) below.

Even though the SFA may be solved analytically, in this Letter we treat the SFA numerically, in the same way as for helium and hydrogen, by solving the Schrödinger equation for the triangular barrier

$$
-\frac{1}{2} \frac{\partial^{2} \chi_{S}}{\partial \eta^{2}}+V_{\mathrm{SFA}}(\eta) \chi_{S}=E \chi_{S},
$$

where $V_{\mathrm{SFA}}(\eta)=-F \eta$ for $\eta>0$ is the part of the potential corresponding to the triangular barrier and $V_{\mathrm{SFA}}(\eta)=2 E$ for $\eta<0$ corresponds to the potential well holding the initial "bound" state. The energy $E$ is equal to the exact resonance energy in the case of hydrogen [see Eq. (S8) for helium]. The equation is solved, similarly to Eq. (6), using an outgoing Airy function (here, an exact solution) to start the integration.

Tunneling times of hydrogen are shown in Fig. 1. Note that all of these times are significantly slower than superluminal, which is less than ten attoseconds (since the barrier width, given approximately by $I_{p} / F$, is less than 50 a. u.). Bohmian tunneling time is, especially for weaker $F$ 's, several orders of magnitude higher than the other tunneling times. This points to the important difference between the time based on Bohmian trajectories and the other definitions of the tunneling times. The Bohmian trajectoriesfollowing the probability density-reflect the time needed by the entire wave function to escape through the barrier, whereas the other times reflect the time needed by each small fraction to pass the barrier. The Bohmian times were therefore found to be related to lifetimes of corresponding resonances: indeed, the agreement between the Bohmian times and lifetimes is nearly perfect. (The resonance lifetimes $\tau_{L}$ were computed from the resonance widths $\Gamma$ taken from Ref. [29] using $\tau_{L}=1 / \Gamma$. Note that for stronger $F$ 's the agreement must necessarily deteriorate since $\Gamma$ stays finite even when the barrier length and the Bohmian tunneling time approaches zero.) Interestingly, the SFA performs substantially worse for Bohmian trajectories than it does for other tunneling times, pointing to much stronger sensitivity of the Bohmian time to the exact shape of the barrier.

As can be seen in Fig. 1, the agreement between the short-range potential approximation (labeled SFA) and the

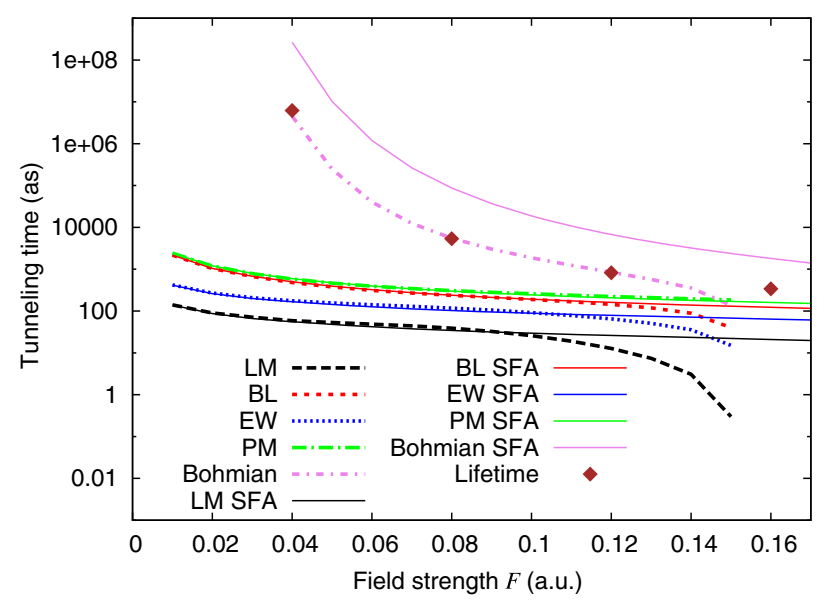

FIG. 1. Tunneling times of hydrogen using the exact parabolic solution, the SFA, and the exact resonance energies. The resonance lifetimes were taken from Ref. [29].

exact solution breaks down as the field strength increases. This can be understood by considering the actual barrier width compared to the width of the triangular potential, $I_{p} / F$, which comes out of the SFA. Specifically, as one approaches the over-the-barrier-ionization (OBI) regime, the actual barrier disappears, while the approximation always predicts a triangular barrier of the width $I_{p} / F$. This also explains why Fig. 2 shows very good agreement between the SFA and the exact solution in helium. For example, helium stays well within the OBI regime for the range of field strengths shown in Fig. 2; hence, the actual barrier shape is close to $I_{p} / F$. In general, we found the four tunneling time definitions to be much less sensitive on the exact barrier shape than ionization probabilities.

A recent work dealing exclusively with the attoclock approach to measuring tunneling time-rather than with the more general theoretical definitions computed heresuggests that the tunneling in hydrogen is instantaneous [17]. However, although Torlina et al. [17] deal extensively with ionization time (corresponding to the time the electron appears at the tunnel exit), no explicit definition of tunneling time is provided or discussed in the paper. Given that the authors of Ref. [17] also question the key "time zero" assumption normally used in the attoclock extraction of tunneling time, without providing an alternative time zero, it seems that the information presented in Ref. [17] is insufficient to definitively conclude instantaneous tunneling (see part IV of the Supplemental Material [26] for more details).

For helium, the time-independent Schrödinger equation is not separable in the parabolic coordinates. Nevertheless, it may be separated approximately using the TIPIS model [30] (see the Supplemental Material [26] for details on computation). The tunneling times of helium plotted in Fig. 2 are, especially for weak fields, relatively similar to those of hydrogen. Mostly owing to a higher lying tunneling ionization threshold, the agreement between 


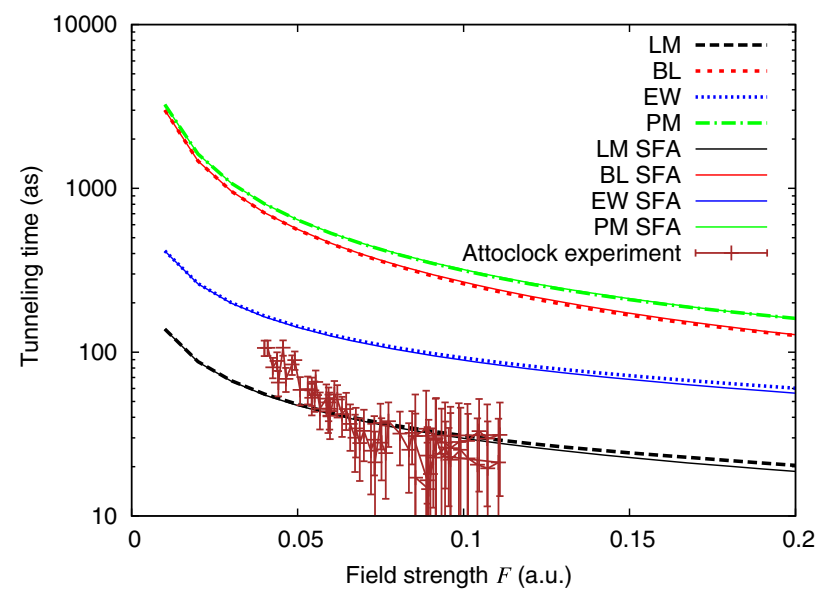

FIG. 2. Tunneling times of helium using TIPIS and the SFA with resonance energies computed using the second-order perturbation theory in comparison with the attoclock experiment.

TIPIS and the SFA is better in helium than in hydrogen. Also, the TIPIS tunneling times go to zero at much higher values of $F$ than for hydrogen-beyond the range studied here. Bohmian times (not shown) are, similar to hydrogen, several orders of magnitude higher than the other tunneling times.

Our numerical results are compared with the recent attoclock experimental data in Fig. 2. The attoclock uses elliptically polarized light to obtain an electron momenta distribution in the plane of polarization, following ionization of the gas [10,31-34]. Tunneling time is experimentally defined as corresponding to the angle of rotation in the electron momenta distribution, relative to what would be expected if the most probable electron trajectory appears at the tunnel exit at the peak of the laser field (for a detailed description of the attoclock concept, see, for example, Ref. [35]). The exact location of the attoclock data points in Fig. 2 may vary up to 100 attoseconds, depending on the calibration method and intensity, hence not excluding superluminal values [17]. Nevertheless, it is clear that, among the approaches to tunneling time discussed here, only Larmor time can be compatible with the attoclock measurements, since the other times lie significantly outside of the experimental range.

It is important to note that the attoclock setup is only able to reliably extract tunneling times that are significantly shorter than the laser period. Hence, for the wavelength of $735 \mathrm{~nm}$ (corresponding to the experimental data plotted in Fig. 2), the attoclock setup would not have been able to reliably access tunneling times significantly longer than 100 attoseconds (see Ref. [15] for an additional explanation). In this case, much longer mid-IR pulses may be a good alternative, particularly since they correspond to a smaller $\gamma$ regime. On the other hand, at sufficiently low frequencies magnetic fields may become important [36,37]. An interesting alternative model that bypasses this

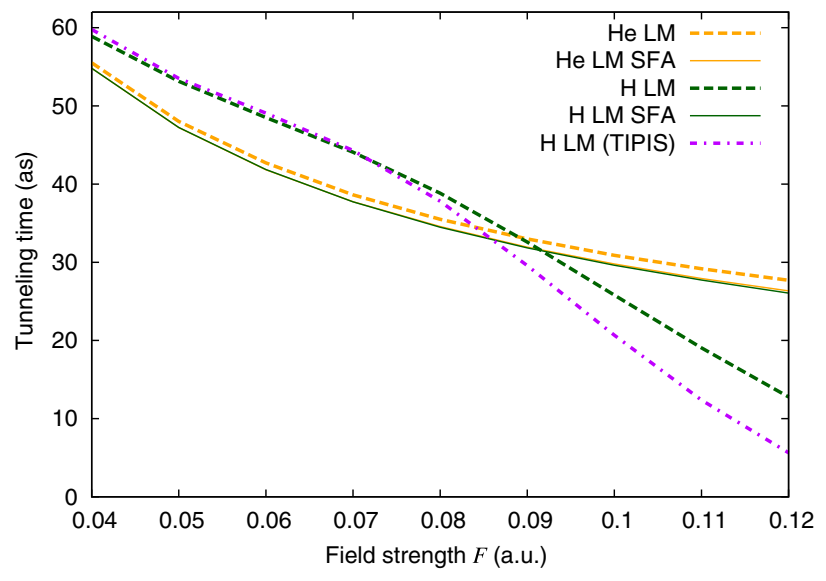

FIG. 3. LM times of hydrogen and helium.

limitation by using a stationary barrier and an extreme ultraviolet pulse to clock the start of the tunneling process (with the IR pulse subsequently used for streaking) was recently proposed in Ref. [15].

Figure 3 compares, in closer detail, the LM times of hydrogen and helium. Interestingly, in the SFA, the LM times of both atoms are almost identical (to the point that it is difficult to discern between them in the figure). Higher resolution of Fig. 3 clearly demonstrates that, in the intensity range studied, the SFA does a better job in the case of helium than in the case of hydrogen. Figure 3 also allows for assessing the effects on accuracy of using the second-order energy and separation constant in TIPIS by comparing it to the exact solution in hydrogen.

Following the Feynman path integral approach, the Larmor time can be expressed [3]

$$
\tau_{\mathrm{LM}}=\operatorname{Re} \frac{\left\langle\psi_{f}|\tau| \psi_{i}\right\rangle}{\left\langle\psi_{f} \mid \psi_{i}\right\rangle}=\operatorname{Re}\left(\tau_{T}^{\Omega}\right)
$$

where $\psi_{i}$ and $\psi_{f}$ correspond to the initial and final states, respectively, and $\tau_{T}^{\Omega}$ is the complex average tunneling time defined within the path integral approach [38]. It is clear from the above definition that the Larmor time corresponds to the weak measurement value of the tunneling time since the weak measurement value of an observable, $a$, is given by $\operatorname{Re}\left(\left\langle\psi_{f}|\hat{a}| \psi_{i}\right\rangle /\left\langle\psi_{f} \mid \psi_{i}\right\rangle\right)$ [12,39].

The significant discrepancy between the Bohmian and Larmor times may be understood by considering that the weak value can differ considerably from the ensemble average obtained using strong value measurements, if this ensemble average is calculated by postselecting only those results for which a later strong measurement reveals the system to be in a state $\left|\psi_{f}\right\rangle$ [12]. This is indeed the case in the attoclock strong field ionization experiment, where tunneling time is extracted from a small fraction of tunneled electrons, all corresponding to the same postselected state, given by $\left|\psi_{f}\right\rangle=\left|p_{0}\right\rangle$, where $p_{0}$ corresponds to the most probable momentum observed at the detector. 
On the other hand, the density of Bohmian trajectories directly represents the quantum probability density. In addition, all Bohmian trajectories representing solutions of the time-independent Schroedinger equation are the same (except for the time shift) and the time they spend in a given region of space is directly proportional to the probability density. Therefore, Bohmian time can be viewed as an ensemble average (as it is weighted by the probability density), and indeed it corresponds to the total ionization time of the state. On the other hand, the Larmor time corresponds to the weak measurement value for the tunneling time [3]. As such, it corresponds to the average over the postselected final state, $\left|p_{0}\right\rangle$. If the fraction of electrons which end up in such a final state $\left|p_{0}\right\rangle$ is very small (corresponding to a low probability of ionization), the Bohmian time can differ considerably from the weak measurement value of the tunneling time. Moreover, since the attoclock experiment intentionally avoids the saturation regime, only a small fraction of the initial bound state wave packet makes it to the detector, thus explaining why it is the Larmor time, rather than the Bohmian time, which may be possible to access with the attoclock.

This research was supported by ETH Research Grant No. ETH-03 09-2 and the Max Planck Center for Attosecond Science (MPC-AS). We acknowledge valuable comments from Ursula Keller, Jan Michael Rost and Ferenc Krausz.

*Corresponding author. landsman@pks.mpg.de

[1] R. Landauer, Nature (London) 341, 567 (1989).

[2] M. Büttiker and R. Landauer, Phys. Rev. Lett. 49, 1739 (1982).

[3] R. Landauer and T. Martin, Rev. Mod. Phys. 66, 217 (1994).

[4] N. Yamada, Phys. Rev. Lett. 93, 170401 (2004).

[5] P. Eckle, A. N. Pfeiffer, C. Cirelli, A. Staudte, R. Dörner, H. G. Muller, M. Büttiker, and U. Keller, Science 322, 1525 (2008).

[6] A. S. Landsman and U. Keller, Phys. Rep. 547, 1 (2015).

[7] C. Spielmann, R. Szipöcs, A. Stingl, and F. Krausz, Phys. Rev. Lett. 73, 2308 (1994).

[8] A. M. Steinberg, P. G. Kwiat, and R. Y. Chiao, Phys. Rev. Lett. 71, 708 (1993).

[9] A. M. Steinberg, Phys. Rev. Lett. 74, 2405 (1995).

[10] A. S. Landsman, M. Weger, J. Maurer, R. Boge, A. Ludwig, S. Heuser, C. Cirelli, L. Gallmann, and U. Keller, Optica 1, 343 (2014).

[11] S. Kocsis, B. Braverman, S. Ravets, M. J. Stevens, R. P. Mirin, L. K. Shalm, and A. M. Steinberg, Science 332, 1170 (2011).

[12] H. M. Wiseman, New J. Phys. 9, 165 (2007).

[13] E. Goulielmakis, Z.-H. Loh, A. Wirth, R. Santra, N. Rohringer, V.S. Yakovlev, S. Zherebtsov, T. Pfeifer,
A. M. Azzeer, M. F. Kling et al., Nature (London) 466, 739 (2010).

[14] A. Wirth, M. T. Hassan, I. Grguraš, J. Gagnon, A. Moulet, T. T. Luu, S. Pabst, R. Santra, Z. A. Alahmed, A. M. Azzeer et al., Science 334, 195 (2011).

[15] R. Pazourek, S. Nagele, and J. Burgdoerfer, Rev. Mod. Phys. 87, 765 (2015).

[16] M. Lein, Nature (London) 485, 313 (2012).

[17] L. Torlina, F. Morales, J. Kaushal, I. Ivanov, A. Kheifets, A. Zielinski, A. Scrinzi, H. G. Muller, S. Sukiasyan, M. Ivanov et al., Nat. Phys. 11, 503 (2015).

[18] E. Pollack and W. Miller, Phys. Rev. Lett. 53, 115 (1984).

[19] EW and PM times will be given more prominence in a follow-up publication, where they will also be related to classical passage time. For the EW and PM times $T=$ $\psi\left(q_{1}\right)-\psi\left(q_{2}\right)$ was used [all other times were calculated as described in the text].

[20] A. Baz', Sov. J. Nucl. Phys. 4, 182 (1967).

[21] E. P. Wigner, Phys. Rev. 98, 145 (1955).

[22] C. Leavens and G. Aers, Solid State Commun. 63, 1101 (1987).

[23] D. Bohm, Phys. Rev. 85, 166 (1952).

[24] C. L. Lopreore and R. E. Wyatt, Phys. Rev. Lett. 82, 5190 (1999).

[25] L. D. Landau and E. M. Lifshitz, Quantum Mechanics: Nonrelativistic Theory (Pergamon Press, New York, 1991).

[26] See Supplemental Material at http://link.aps.org/ supplemental/10.1103/PhysRevLett.116.233603 for details on computation and the attoclock concept.

[27] L. Keldysh, J. Exp. Theor. Phys. 20, 1307 (1965).

[28] R. Murray, W.-K. Liu, and M. Ivanov, Phys. Rev. A 81, 023413 (2010).

[29] R. J. Damburg and V. V. Kolosov, J. Phys. B 9, 3149 (1976).

[30] A. N. Pfeiffer, C. Cirelli, M. Smolarski, D. Dimitrovski, M. Abu-samha, L. B. Madsen, and U. Keller, Nat. Phys. 8, 76 (2012).

[31] A. S. Landsman, C. Hofmann, A. N. Pfeiffer, C. Cirelli, and U. Keller, Phys. Rev. Lett. 111, 263001 (2013).

[32] C. Hofmann, A. S. Landsman, C. Cirelli, A. N. Pfeiffer, and U. Keller, J. Phys. B 46, 125601 (2013).

[33] R. Boge, C. Cirelli, A. S. Landsman, S. Heuser, A. Ludwig, J. Maurer, M. Weger, L. Gallmann, and U. Keller, Phys. Rev. Lett. 111, 103003 (2013).

[34] A. S. Landsman and U. Keller, J. Phys. B 47, 204024 (2014).

[35] L. Gallmann, C. Cirelli, and U. Keller, Annu. Rev. Phys. Chem. 63, 447 (2012).

[36] A. S. Landsman, S. A. Cohen, and A. H. Glasser, Phys. Rev. Lett. 96, 015002 (2006).

[37] A. Ludwig, J. Maurer, B. W. Mayer, C. R. Phillips, L. Gallmann, and U. Keller, Phys. Rev. Lett. 113, 243001 (2014).

[38] D. Sokolovski and L. M. Baskin, Phys. Rev. A 36, 4604 (1987).

[39] Y. Aharonov, D. Z. Albert, and L. Vaidman, Phys. Rev. Lett. 60, 1351 (1988). 